\title{
Affine and Deformable Registration Based on Polynomial Expansion
}

\author{
Gunnar Farnebäck and Carl-Fredrik Westin \\ Department of Radiology, Brigham and Womens' Hospital, \\ Harvard Medical School, Boston, MA 02115, USA
}

\begin{abstract}
This paper presents a registration framework based on the polynomial expansion transform. The idea of polynomial expansion is that the image is locally approximated by polynomials at each pixel. Starting with observations of how the coefficients of ideal linear and quadratic polynomials change under translation and affine transformation, algorithms are developed to estimate translation and compute affine and deformable registration between a fixed and a moving image, from the polynomial expansion coefficients. All algorithms can be used for signals of any dimensionality. The algorithms are evaluated on medical data.
\end{abstract}

\section{Introduction}

Medical images are brought into spatial correspondence, or aligned, by the use of registration algorithms. Nonrigid registration refers to the set of techniques that allow the alignment of datasets that are mismatched in a nonrigid, or nonuniform manner. Such misalignments can result from physical deformation processes, or can be a result of morphological variability. Nonrigid deformation is also characteristic of the organs and soft tissues of the abdomen and pelvis. In addition, nonrigid morphological differences can arise when comparisons are made among image datasets acquired from different individuals. These changes can be a result of normal anatomical variability or the product of pathological processes. Because the gross structure of the brain is essentially similar among humans (and even among related species), the factors described above tend to produce local nonrigid shape differences. Nonrigid brain registration techniques have numerous applications. They have been used to align scans of different brains, permitting the characterization of normal and pathological morphological variation (brain mapping). They have also been used to align anatomical templates with specific datasets, thus facilitating segmentation (i.e. segmentation by registration). More recently, these techniques have been used to capture changes which occur during neurosurgery. With the ongoing development of robust algorithms and advanced hardware platforms, further applications in surgical visualization and enhanced functional image analysis are inevitable.

Spatial normalization in clinical studies involves the registration of images and the generation of a stereotaxic atlas that represents the statistical distribution of the group at each voxel 12234 . 


\section{Polynomial Expansion}

The idea of polynomial expansion is to approximate the signal values in some neighborhood of each pixel with a polynomial. In this paper we are only interested in using linear or quadratic polynomials. In the quadratic case we have the local signal model, expressed in a local coordinate system,

$$
f(\mathbf{x}) \sim \mathbf{x}^{T} \mathbf{A} \mathbf{x}+\mathbf{b}^{T} \mathbf{x}+c,
$$

where $\mathbf{A}$ is a symmetric matrix, $\mathbf{b}$ a vector and $c$ a scalar. In the linear case we drop the first term and instead have the local signal model

$$
f(\mathbf{x}) \sim \mathbf{b}^{T} \mathbf{x}+c .
$$

The coefficients are determined from a weighted least squares fit to the signal values in the neighborhood. The weighting has two factors called certainty and applicability. These terms are the same as in normalized convolution 5. The certainty is coupled to the signal values in the neighborhood. For example it is generally a good idea to set the certainty to zero outside the image. Then neighborhood points outside the image have no impact on the coefficient estimation.

The applicability determines the relative weight of points in the neighborhood based on their position in the neighborhood. Typically one wants to give most weight to the center point and let the weights decrease radially. In this paper we only use Gaussian applicability, both to simplify the presentation and because its Cartesian separability and isotropy are valuable features. This also allows us to describe the scale of the polynomial expansion with a single value, its standard deviation $\sigma$. This is directly related to the scale of the structures which will be captured by the expansion coefficients. Thus a small $\sigma$ can be used to capture fine details and a large $\sigma$ can be used to capture coarse details. More details about polynomial expansion can be found in [6].

\section{Translation Estimation}

For translation estimation we assume that the whole signal has been globally translated. As a preparation we start by analyzing what happens if a single quadratic polynomial is translated. First consider the quadratic polynomial $f_{\text {fixed }}(\mathbf{x})$ and construct a new signal $f_{\text {moving }}$ by a global translation by $\mathbf{d}$,

$$
\begin{aligned}
& f_{\text {fixed }}(\mathbf{x})=\mathbf{x}^{T} \mathbf{A}_{\mathrm{f}} \mathbf{x}+\mathbf{b}_{\mathrm{f}}^{T} \mathbf{x}+c_{\mathrm{f}}, \\
& f_{\text {moving }}(\mathbf{x})=f_{\text {fixed }}(\mathbf{x}-\mathbf{d})=(\mathbf{x}-\mathbf{d})^{T} \mathbf{A}_{\mathrm{f}}(\mathbf{x}-\mathbf{d})+\mathbf{b}_{\mathrm{f}}^{T}(\mathbf{x}-\mathbf{d})+c_{\mathrm{f}} \\
& =\mathbf{x}^{T} \mathbf{A}_{\mathrm{f}} \mathbf{x}+\left(\mathbf{b}_{\mathrm{f}}-2 \mathbf{A}_{\mathrm{f}} \mathbf{d}\right)^{T} \mathbf{x}+\mathbf{d}^{T} \mathbf{A}_{\mathrm{f}} \mathbf{d}-\mathbf{b}_{\mathrm{f}}^{T} \mathbf{d}+c_{\mathrm{f}}=\mathbf{x}^{T} \mathbf{A}_{\mathrm{m}} \mathbf{x}+\mathbf{b}_{\mathrm{m}}^{T} \mathbf{x}+c_{\mathrm{m}} .
\end{aligned}
$$

Equating the quadratic and linear coefficients yields

$$
\begin{aligned}
\mathbf{A}_{\mathrm{m}} & =\mathbf{A}_{\mathrm{f}}, \\
\mathbf{b}_{\mathrm{m}} & =\mathbf{b}_{\mathrm{f}}-2 \mathbf{A}_{\mathrm{f}} \mathbf{d} .
\end{aligned}
$$


If $\mathbf{A}_{\mathrm{f}}$ is non-singular, (6) alone lets us solve for the translation $\mathbf{d}$, regardless of signal dimensionality,

$$
\mathbf{d}=\frac{1}{2} \mathbf{A}_{\mathrm{f}}^{-1}\left(\mathbf{b}_{\mathrm{f}}-\mathbf{b}_{\mathrm{m}}\right)
$$

In reality we do not have a single global polynomial but after polynomial expansion of the signal we get one polynomial approximation of the signal at each pixel. Let $\mathbf{A}_{\mathrm{f}}(\mathbf{x}), \mathbf{b}_{\mathrm{f}}(\mathbf{x})$, and $c_{\mathrm{f}}(\mathbf{x})$ be the quadratic polynomial expansion coefficients for the fixed image and $\mathbf{A}_{\mathrm{m}}(\mathbf{x}), \mathbf{b}_{\mathrm{m}}(\mathbf{x})$, and $c_{\mathrm{m}}(\mathbf{x})$ be the coefficients for the moving image. According to (5) we should have $\mathbf{A}_{\mathrm{f}}(\mathbf{x})=\mathbf{A}_{\mathrm{m}}(\mathbf{x})$ but we cannot expect this to hold when the signal no longer is a single polynomial. Instead we use their average and introduce

$$
\mathbf{A}(\mathbf{x})=\frac{\mathbf{A}_{\mathrm{f}}(\mathbf{x})+\mathbf{A}_{\mathrm{m}}(\mathbf{x})}{2}, \quad \Delta \mathbf{b}(\mathbf{x})=\frac{1}{2}\left(\mathbf{b}_{\mathrm{f}}(\mathbf{x})-\mathbf{b}_{\mathrm{m}}(\mathbf{x})\right)
$$

to transform (6) into the primary constraint

$$
\mathbf{A}(\mathbf{x}) \mathbf{d}=\Delta \mathrm{b}(\mathrm{x})
$$

One possible approach now is to solve (9) pointwise and average the translation vectors over the image to estimate the global translation $\mathbf{d}$. This is not a good solution, however, since errors in $\mathbf{A}(\mathbf{x})$ and $\Delta \mathbf{b}(\mathbf{x})$ (caused by noise and model imperfections) become unnecessarily amplified in the pointwise computations. Instead we compute $\mathbf{d}$ by minimizing the squared error in the constraints over the whole image,

$$
\epsilon^{2}=\sum_{\mathbf{x}}\|\mathbf{A}(\mathbf{x}) \mathbf{d}-\Delta \mathbf{b}(\mathbf{x})\|^{2}
$$

giving the least squares solution

$$
\mathbf{d}=\left(\sum \mathbf{A}(\mathbf{x})^{T} \mathbf{A}(\mathbf{x})\right)^{-1} \sum \mathbf{A}(\mathbf{x})^{T} \boldsymbol{\Delta} \mathbf{b}(\mathbf{x}) .
$$

\subsection{Incorporating a Priori Knowledge}

We started the derivation of (11) by considering a translation of a single polynomial. In that case (5) and (6) are valid regardless how large the translation is. For a real signal the differences in the polynomial coefficients at any given pixel between the fixed and the moving images are only partially explained by these equations. The second factor is that the polynomial approximations of the signal vary spatially as well. The larger the distance, the less similar the approximating polynomials can be expected to be. Thus the constraint (9) can be expected to be more uncertain the larger the translation.

Fortunately we are not restricted to comparing two polynomials at the same coordinate. If we happen to have some a priori knowledge about the translation we can instead compare the polynomial coefficients at $\mathbf{x}$ in the fixed image to the 
coefficients at $\mathbf{x}+\tilde{\mathbf{d}}$ in the moving image, where $\tilde{\mathbf{d}}$ is the rounded a priori estimate. Then we effectively only need to estimate the relative translation between the true translation and $\tilde{\mathbf{d}}$, which hopefully is smaller. More precisely we can include this observation in the algorithm by replacing (8) by

$$
\begin{aligned}
\mathbf{A}(\mathbf{x}) & =\frac{\mathbf{A}_{\mathrm{f}}(\mathbf{x})+\mathbf{A}_{\mathrm{m}}(\tilde{\mathbf{x}})}{2}, \\
\Delta \mathbf{b}(\mathbf{x}) & =\frac{1}{2}\left(\mathbf{b}_{\mathrm{f}}(\mathbf{x})-\mathbf{b}_{\mathrm{m}}(\tilde{\mathbf{x}})\right)+\mathbf{A}(\mathbf{x}) \tilde{\mathbf{d}}(\mathbf{x}), \\
\tilde{\mathbf{x}} & =\mathbf{x}+\tilde{\mathbf{d}}(\mathbf{x}) .
\end{aligned}
$$

We let $\tilde{\mathbf{d}}$ be space variant because we will need it later and also to allow us to avoid getting any $\tilde{\mathbf{x}}$ outside the border of the image.

\subsection{Iterative and Multi-scale Translation Estimation}

A consequence of the inclusion of an a priori translation estimate in the algorithm is that we can close the loop and iterate. A better a priori estimate means a smaller relative translation, which in turn improves the chances for a good new translation estimate. This can be done within one scale or between scales. In the first case we keep the polynomial expansion coefficients between the iterations and only recompute (14), (12), (13), and (11). In most cases this converges in only a few iterations, although not necessarily to a correct result if the translation is too large. Notice that since we are rounding the translation estimates at the start of each iteration we are very unlikely to get into any small scale oscillations. The initial translation estimate in the first step can be set to zero, unless actual knowledge about it is available.

\subsection{Weighting the Constraints}

As already has been discussed, the constraint (9) tends to become more uncertain the larger the translation is. One way to estimate this uncertainty is to see how much $\mathbf{A}_{\mathrm{m}}(\tilde{\mathbf{x}})$ differs from $\mathbf{A}_{\mathrm{f}}(\mathbf{x})$, since they according to (5) should ideally be equal. Thus we introduce

$$
\gamma_{2}(\mathbf{x})=e^{-\frac{\alpha\left\|\mathbf{A}_{\mathbf{f}}(\mathbf{x})-\mathbf{A}_{\mathrm{m}}(\tilde{\mathbf{x}})\right\|_{\mathrm{F}}^{2}}{\left\|\mathbf{A}_{\mathbf{f}}(\mathbf{x})\right\|_{\mathrm{F}}^{2}+\left\|\mathbf{A}_{\mathrm{m}}(\tilde{\mathbf{x}})\right\|_{\mathrm{F}}^{2}}},
$$

which will vary from 1 when $\mathbf{A}_{\mathrm{m}}(\tilde{\mathbf{x}})$ and $\mathbf{A}_{\mathrm{f}}(\mathbf{x})$ are equal down to $e^{-2 \alpha}$ when they are opposite. The squared Frobenius norm $\|\cdot\|_{\mathrm{F}}^{2}$ is given by the sum of the squared matrix elements. Now we introduce $\gamma_{2}(\mathbf{x})$ as a weight in the squared error measure (10), by once more modifying the definition of $\mathbf{A}(\mathbf{x})$ and $\Delta \mathbf{b}(\mathbf{x})$, replacing (12) and (13) by

$$
\begin{aligned}
\mathbf{A}(\mathbf{x}) & =\gamma_{2}(\mathbf{x}) \frac{\mathbf{A}_{\mathrm{f}}(\mathbf{x})+\mathbf{A}_{\mathrm{m}}(\tilde{\mathbf{x}})}{2}, \\
\Delta \mathbf{b}(\mathbf{x}) & =\gamma_{2}(\mathbf{x})\left(\frac{1}{2}\left(\mathbf{b}_{\mathrm{f}}(\mathbf{x})-\mathbf{b}_{\mathrm{m}}(\tilde{\mathbf{x}})\right)+\mathbf{A}(\mathbf{x}) \tilde{\mathbf{d}}(\mathbf{x})\right) .
\end{aligned}
$$




\subsection{Using Linear Polynomial Expansion}

We can repeat these derivations using the linear polynomial model (2), instead of the quadratic model (10). This changes (3) and (4) into

$$
\begin{aligned}
f_{\text {fixed }}(\mathbf{x}) & =\mathbf{b}_{\mathrm{f}}^{T} \mathbf{x}+c_{\mathrm{f}} \\
f_{\text {moving }}(\mathbf{x}) & =f_{\text {fixed }}(\mathbf{x}-\mathbf{d})=\mathbf{b}_{\mathrm{f}}^{T} \mathbf{x}+c_{\mathrm{f}}-\mathbf{b}_{\mathrm{f}}^{T} \mathbf{d}=\mathbf{b}_{\mathrm{m}}^{T} \mathbf{x}+c_{\mathrm{m}} .
\end{aligned}
$$

Equating the coefficients now gives

$$
\mathbf{b}_{\mathrm{m}}=\mathbf{b}_{\mathrm{f}}, \quad c_{\mathrm{m}}=c_{\mathrm{f}}-\mathbf{b}_{\mathrm{f}}^{T} \mathbf{d},
$$

and the correspondences to (15)-17) become

$$
\begin{aligned}
\gamma_{1}(\mathbf{x}) & =e^{-\frac{\alpha\left\|\mathbf{b}_{\mathrm{f}}(\mathbf{x})-\mathbf{b}_{\mathrm{m}}(\tilde{\mathbf{x}})\right\|_{\mathrm{F}}^{2}}{\left\|\mathbf{b}_{\mathrm{f}}(\mathbf{x})\right\|_{\mathrm{F}}^{2}+\left\|\mathbf{b}_{\mathrm{m}}(\tilde{\mathbf{x}})\right\|_{\mathrm{F}}^{2}}}, \\
\mathbf{b}(\mathbf{x}) & =\gamma_{1}(\mathbf{x}) \frac{\mathbf{b}_{\mathrm{f}}(\mathbf{x})+\mathbf{b}_{\mathrm{m}}(\tilde{\mathbf{x}})}{2} \\
\Delta c(\mathbf{x}) & =\gamma_{1}(\mathbf{x})\left(\mathbf{c}_{\mathrm{f}}(\mathbf{x})-\mathbf{c}_{\mathrm{m}}(\tilde{\mathbf{x}})+\mathbf{b}(\mathbf{x})^{T} \tilde{\mathbf{d}}(\mathbf{x})\right) .
\end{aligned}
$$

Now we add the linear constraint into (10), using $\beta_{1}$ and $\beta_{2}$ as weights for the linear and quadratic parts,

$$
\epsilon^{2}=\sum_{\mathbf{x}} \beta_{1}\left(\mathbf{b}(\mathbf{x})^{T} \mathbf{d}-\Delta c(\mathbf{x})\right)^{2}+\beta_{2}\|\mathbf{A}(\mathbf{x}) \mathbf{d}-\Delta \mathbf{b}(\mathbf{x})\|^{2},
$$

with the least squares solution

$$
\begin{aligned}
& \mathbf{G}=\sum \beta_{1} \mathbf{b}(\mathbf{x}) \mathbf{b}(\mathbf{x})^{T}+\beta_{2} \mathbf{A}(\mathbf{x})^{T} \mathbf{A}(\mathbf{x}), \\
& \mathbf{h}=\sum \beta_{1} \mathbf{b}(\mathbf{x}) \Delta c(\mathbf{x})+\beta_{2} \mathbf{A}(\mathbf{x})^{T} \Delta \mathbf{b}(\mathbf{x}), \\
& \mathbf{d}=\mathbf{G}^{-1} \mathbf{h} .
\end{aligned}
$$

\section{Affine Registration}

Now we turn to the more interesting case of affine registration and to simplify the presentation we do this in 2D. Generalizations to higher dimensionalities are straightforward. The deformation field is now space variant

$$
\begin{aligned}
& d_{x}(x, y)=a_{1} x+a_{2} y+a_{5}, \\
& d_{y}(x, y)=a_{3} x+a_{4} y+a_{6},
\end{aligned}
$$

and we can rewrite this in matrix form as

$$
\begin{aligned}
\mathbf{d}(\mathbf{x}) & =\mathbf{S}(\mathbf{x}) \mathbf{p} \\
\mathbf{S}(\mathbf{x}) & =\left(\begin{array}{llllll}
x & y & 0 & 0 & 1 & 0 \\
0 & 0 & x & y & 0 & 1
\end{array}\right), \\
\mathbf{p} & =\left(\begin{array}{llllll}
a_{1} & a_{2} & a_{3} & a_{4} & a_{5} & a_{6}
\end{array}\right)^{T} .
\end{aligned}
$$


To estimate the parameters of the affine deformation field is as simple as combining (24) and (29),

$$
\epsilon^{2}=\sum_{\mathbf{x}} \beta_{1}\left(\mathbf{b}(\mathbf{x})^{T} \mathbf{S}(\mathbf{x}) \mathbf{p}-\Delta c(\mathbf{x})\right)^{2}+\beta_{2}\|\mathbf{A}(\mathbf{x}) \mathbf{S}(\mathbf{x}) \mathbf{p}-\Delta \mathbf{b}(\mathbf{x})\|^{2}
$$

and we get the parameters $\mathbf{p}$ from the least squares solution

$$
\begin{aligned}
\mathbf{G} & =\sum \beta_{1} \mathbf{S}_{i}^{T} \mathbf{b}_{i} \mathbf{b}_{i}^{T} \mathbf{S}_{i}+\beta_{2} \mathbf{S}_{i}^{T} \mathbf{A}_{i}^{T} \mathbf{A}_{i} \mathbf{S}_{i}, \\
\mathbf{h} & =\sum \beta_{1} \mathbf{S}_{i}^{T} \mathbf{b}_{i} \Delta c_{i}+\beta_{2} \mathbf{S}_{i} \mathbf{A}_{i}^{T} \Delta \mathbf{b}_{i}, \\
\mathbf{p} & =\mathbf{G}^{-1} \mathbf{h},
\end{aligned}
$$

where we have replaced the spatial coordinate by a subscript to shorten the notation. We can notice that this approach not only works for affine deformation but for any parametric deformation field which is linear in its parameters.

\section{Deformable Registration}

We can derive a deformable registration algorithm if we relax the assumption about a global translation or a globally affine deformation, and instead do the summation in either of (24) or (32) over a neighborhood around each pixel, thereby getting an estimate for each pixel. For computational reasons we will only work with local translations. This does not impose a strong structure on the deformation field, but due to lowpass filtering in the algorithm, the deformation field automatically becomes smooth. More exactly we change (24) to

$\epsilon^{2}(\mathbf{x})=\sum_{\mathbf{y}} w(\mathbf{y})\left(\beta_{1}\left(\mathbf{b}(\mathbf{x}-\mathbf{y})^{T} \mathbf{d}(\mathbf{x})-\Delta c(\mathbf{x}-\mathbf{y})\right)^{2}+\beta_{2}\|\mathbf{A}(\mathbf{x}-\mathbf{y}) \mathbf{d}(\mathbf{x})-\boldsymbol{\Delta} \mathbf{b}(\mathbf{x}-\mathbf{y})\|^{2}\right)$,

where $w$ weights the points in the neighborhood around each pixel. This weight can be any lowpass function but here we assume it to be Gaussian. Clearly this equation can be interpreted as a convolution of the pointwise contributions to the squared error (24) with the lowpass filter $w$. The solution is

$$
\begin{aligned}
\mathbf{G}(\mathbf{x}) & =\beta_{1} \mathbf{b}(\mathbf{x}) \mathbf{b}(\mathbf{x})^{T}+\beta_{2} \mathbf{A}(\mathbf{x})^{T} \mathbf{A}(\mathbf{x}), \\
\mathbf{h}(\mathbf{x}) & =\beta_{1} \mathbf{b}(\mathbf{x}) \Delta c(\mathbf{x})+\beta_{2} \mathbf{A}(\mathbf{x})^{T} \boldsymbol{\Delta} \mathbf{b}(\mathbf{x}), \\
\mathbf{G}_{\mathrm{avg}}(\mathbf{x}) & =(\mathbf{G} * w)(\mathbf{x}), \\
\mathbf{h}_{\mathrm{avg}}(\mathbf{x}) & =(\mathbf{h} * w)(\mathbf{x}), \\
\mathbf{d}(\mathbf{x}) & =\mathbf{G}_{\mathrm{avg}}(\mathbf{x})^{-1} \mathbf{h}_{\mathrm{avg}}(\mathbf{x}) .
\end{aligned}
$$

\section{$6 \quad$ Experiments}

The affine and deformable registration algorithms have been implemented in Matlab and in $\mathrm{C}++$ using the Insight Toolkit (ITK) 7. Both implementations have similar performance and have been tested on a Pentium IV at $2.8 \mathrm{GHz}$. The time measurements in this section are for the $\mathrm{C}++$ implementation. 


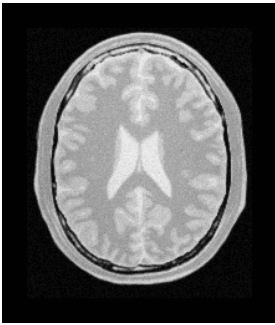

(a) original

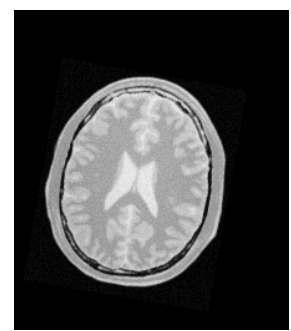

(b) transformed

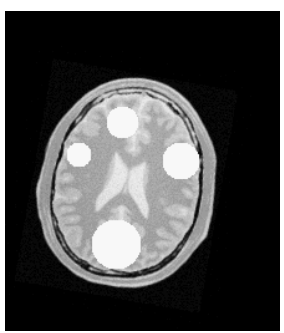

(c) blobs inserted

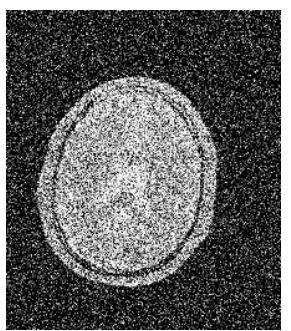

(d) noise added

Fig. 1. Brain images from $\operatorname{ITK}(\mathrm{a}, \mathrm{b})$ and corrupted versions (c, d)

\subsection{Affine Registration}

The affine registration has been tested in $2 \mathrm{D}$ on example data from ITK. Figure 1 (a) shows a brain image. This has been translated, rotated by $10^{\circ}$ and scaled down by a factor 1.2 in Fig. 1(b). The affine registration algorithm takes 3.0 seconds and gives affine parameters corresponding to a rotation of $9.998^{\circ}$ and a minimum and maximum scaling of 1.1991 and 1.1999 respectively. As a comparison the ITK example code for affine registration, ImageRegistration9, takes 28 seconds and gives a rotation estimate of $9.68^{\circ}$ and scalings 1.1994 and 1.2023 .

To test the sensitivity to disturbances, we have inserted a number of blobs in the second image, giving the image shown in Fig. 1(c). In Fig. 1(d) we have the result of adding a substantial amount of (truncated) Gaussian noise. Our algorithm for (c) takes 3.0 seconds and gives a rotation angle of $9.989^{\circ}$ and for (d) it takes 4.5 seconds and gives an angle of $10.24^{\circ}$. The ITK example code takes 35 seconds and gives an angle of $9.22^{\circ}$ for (c) and 53 seconds and $9.08^{\circ}$ for (d). The scaling parameters are at most off by $0.5 \%$ in all cases. The differences in time is a question of a varying number of iterations for both methods.

\subsection{Deformable Registration}

The deformable registration algorithm has been tested in 3D on pig CT data (spatial resolution $1 \times 1 \times 3 \mathrm{~mm}$ ) in a segmentation by registration experiment. This

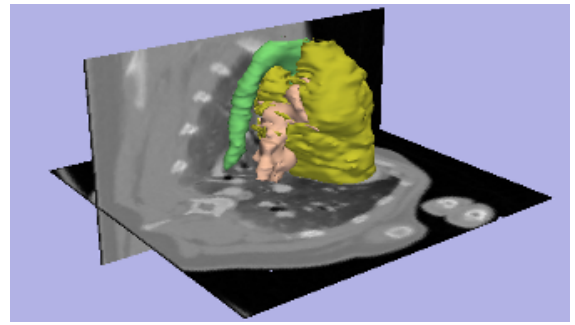

(a) proposed algorithm

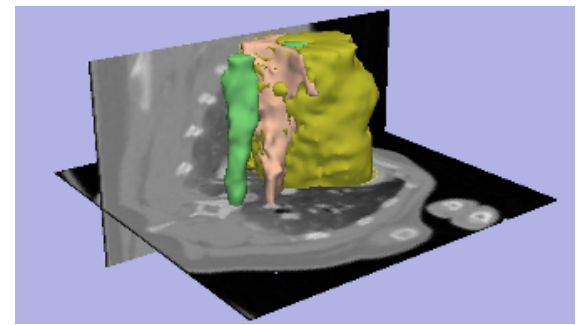

(b) demons algorithm

Fig. 2. Warped pig organs 
involves one data set where a few organs have been hand-segmented and a second data set where no segmentation is known. The CT volumes were registered using the deformable registration algorithm to obtain a deformation field, which was then used to warp the segmentation over from the first data set to the second. Figure 2(a) shows the warped segmentations superimposed on the second volume. For comparison Fig. 2(b) shows the corresponding result when the ITK implementation of demons algorithm (example code DeformableRegistration2 converted to 3D) was used. The anatomy is poorly aligned in the latter case and it is clear that our algorithm performed more robustly than the demons algorithm in this example. The deformable registrations were preceded by affine registration with corresponding parameters.

\section{Conclusions}

We have presented a new registration framework and derived algorithms for translation estimation, affine registration, and deformable registration. All algorithms are valid for any dimensionality. Experiments on medical data indicate that the affine registration is both fast and accurate, and that the deformable registration performs robustly.

\section{Acknowledgments}

This work was supported by grants from the DARPA, executed by the U.S. Army Medical Research and Materiel Command/TATRC Cooperative Agreement, Contract \# W81XWH-04-2-0012, and NIH P41-RR13218.

\section{References}

1. Mazziotta, J.C., Toga, A.W., Evans, A.C., Fox, P., Lancaster, J.L.: A probabilistic atlas of the human brain: Theory and rationale for its development. Neuroimage 2(2) (1995) 89-101

2. Thompson, P.M., Toga, A.W.: Detection, visualization and animation of abnormal anatomic structure with a deformable probabilistic atlas based on random vector field transformations. Medical Image Analysis 1(4) (1997) 271-294

3. Grenander, U., Miller, M.: Computational anatomy: an emerging discipline. Q. Appl. Math. 56(4) (1998) 617-694

4. Guimond, A., Meunier, J., Thirion, J.P.: Average brain models: a convergence study. Comput. Vision Imag. Understand. 77(2) (2000) 192-210

5. Knutsson, H., Westin, C.F.: Normalized and differential convolution: Methods for interpolation and filtering of incomplete and uncertain data. In: Proceedings of IEEE Computer Society Conference on Computer Vision and Pattern Recognition, New York City, USA, IEEE (1993) 515-523

6. Farnebäck, G.: Polynomial Expansion for Orientation and Motion Estimation. PhD thesis, Linköping University, SE-581 83 Linköping, Sweden (2002) Dissertation No 790, ISBN 91-7373-475-6.

7. ITK: The Insight Segmentation and Registration Toolkit, http://www.itk.org 